\title{
ENHANCEMENT OF NEUTRALIZATION REACTION IN COLLOIDAL FERRIC HYDROUS OXIDE $\dagger$
}

\author{
Akos Vertes $\ddagger$ \\ Radiation Laboratory, University of Notre Dame, Notre Dame, IN 46556, U.S.A.
}

(Received 11 February 1985; in revised form 3 April 1985)

\begin{abstract}
Neutralization reaction was investigated in negative ferric hydrous oxide sols. The adsorption isotherm of $\mathrm{OH}^{-}$ions on the surface of colloidal particles was measured to provide the variation of their charge with $\mathrm{pH}$. Impulse radiolysis generated the reacting ions in the sol. Using a fast DC conductivity setup for detection, a significant increase in the neutralization rate coefficient was found at low $\mathrm{OH}^{-}$concentrations. The phenomenon was discussed in terms of the theory of diffusion controlled reactions for particles interacting with Coulomb and Yukawa potential.
\end{abstract}

\section{INTRODUCTION}

TRANSPORT OF corrosion products in the primary cooling system of water cooled nuclear reactors has drawn attention to the behavior of iron oxide colloids undergoing irradiation. ${ }^{(1)}$ Suspensions of other fissile oxides had been examined earlier. ${ }^{(2)}$ The basic question these investigations have posed is how activated corrosion products travel around in the system and how sedimentation terminates their trip causing contamination. Both of these investigations were performed under stationary conditions; dissolution ${ }^{(1)}$ and fragmentation ${ }^{(2)}$ effects were found to be caused by substantial doses.

The search for candidates for water splitting electrodes led to experiments with colloidal metal "microelectrodes"- -see, e.g. Refs. $(3,4)$-and recently with colloidal oxide semiconductors ${ }^{(5,6)}$ such as $\mathrm{TiO}_{2}$ and $\alpha-\mathrm{Fe}_{2} \mathrm{O}_{3}$ dispersions. The suspected advantage inherent in the small particle size, besides their enormous surface, is the possibility of avoiding recombination of light generated electrons and holes before these reach the surface. This of course could improve the yield of water cleavage systems.

Our aim was to explore fast processes immediately after irradiation in ferric hydrous oxide sols in order to understand the fate of water radiolysis products in such systems. The contribution of these processes to radiation induced coagulation of iron oxide colloids and to the consequent contamination of the primary cooling circuit of nuclear power plants may more clearly be understood in this way.

$\dagger$ The research described herein was supported by the Office of Basic Energy Sciences of the Department of Energy.

$\$$ Present address: Central Research Institute for Physics P. O. Box 49, H-1525 Budapest, Hungary.
The present paper deals with the effect of negatively charged colloidal particles on the rate of the neutralization reaction

$$
\mathrm{H}^{+}+\mathrm{OH}^{-} \stackrel{k_{1}}{\longrightarrow} \mathrm{H}_{2} \mathrm{O}
$$

where $k_{1}$ is the rate coefficient. This effect is originated in the possibility of a new reaction, namely the reaction of $\mathrm{H}^{+}$ions with $\mathrm{OH}^{-}$ions adsorbed on the surface of colloidal particles. Since the charge of the colloidal particle depends on the bulk concentration of $\mathrm{OH}^{-}$ions, $c_{\mathrm{OH}^{-}}$, the effective neutralization rate coefficient, $k_{\text {eff }}$, will be a function of this concentration.

In order to characterize the colloid as a reactant we needed the relation between the bulk concentration of $\mathrm{OH}^{-}$and the charge of individual particles. The measurement of adsorption isotherms of hydroxide ions on the surface provided information on the charge of single particles. The second step was to measure the effective rate coefficient of the decay of radiation generated hydrogen ions in a wide $c_{\mathrm{OH}}-$ range. This was made possible by the nanosecond conductivity setup of Janata. ${ }^{(7)}$

In the Discussion we invoke the theory of diffusion controlled reactions to describe our experimental findings.

\section{EXPERIMENTAL}

2.1. Preparation and characterization of the sols

Negative ferric hydrous oxide sols ${ }^{(8)}$ were prepared by the method of Powis ${ }^{(9)}: 200 \mathrm{~cm}^{3}$ of a solution $0.0033 \mathrm{M}$ in $\mathrm{FeCl}_{3}$ and $0.0015 \mathrm{M}$ in $\mathrm{HCl}$ was added gradually to $300 \mathrm{~cm}^{3}$ of well stirred $0.016 \mathrm{M}$ $\mathrm{KOH}$. The solutions were kept under $\mathrm{N}_{2}$ atmosphere and at $\mathrm{O}^{\circ} \mathrm{C}$ before and during the mixing. 
Keeping the sol in the $\mathrm{N}_{2}$ atmosphere provided stability for at least one week. Samples for experiments were diluted from $30 \mathrm{~cm}^{3}$ parts of this sol to $480 \mathrm{~cm}^{3}$ with $\mathrm{N}_{2}$ saturated triple distilled water. All other solutions were prepared with the same water and from Baker Analysed Reagents. The samples were allowed to age for one hour before experiments. Although other methods of preparation ${ }^{(10,11)}$ offered monodisperse $\alpha-\mathrm{Fe}_{2} \mathrm{O}_{3}$ sols, their particle size turned out to be too large.

We measured particle size by light scattering which indicated $100 \AA$ for their radius. No considerable aggregation effect was detected during the first day after preparation.

The total potassium concentration was checked with a potassium electrode; $\mathrm{pH}$ and electrical conductivity were monitored during titration with $0.0097 \mathrm{M} \mathrm{HCl}$. The adsorption isotherm calculated on this basis is displayed in Fig. 1. A Langmuir isotherm was fitted to the measured points:

$$
K c_{\mathrm{OH}^{-}}=\theta /(1-\theta) \text {, }
$$

here $\theta=c_{\mathrm{OH}^{-}}^{\text {ads }} / c_{\mathrm{OH}^{-}}^{\text {adsmax }}, K$ and $c_{\mathrm{OH}^{-}}^{\text {adsmax }}$ are the two parameters of the curve denoting the ratio of adsorption and desorption rate coefficients and maximum adsorbed $\mathrm{OH}^{-}$concentration, respectively. The actual $\mathrm{OH}^{-}$concentration on the surface is

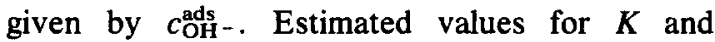
$c_{\mathrm{OH}^{-}}^{\text {adsmax }}$ can be obtained by curve fitting. In a colloid

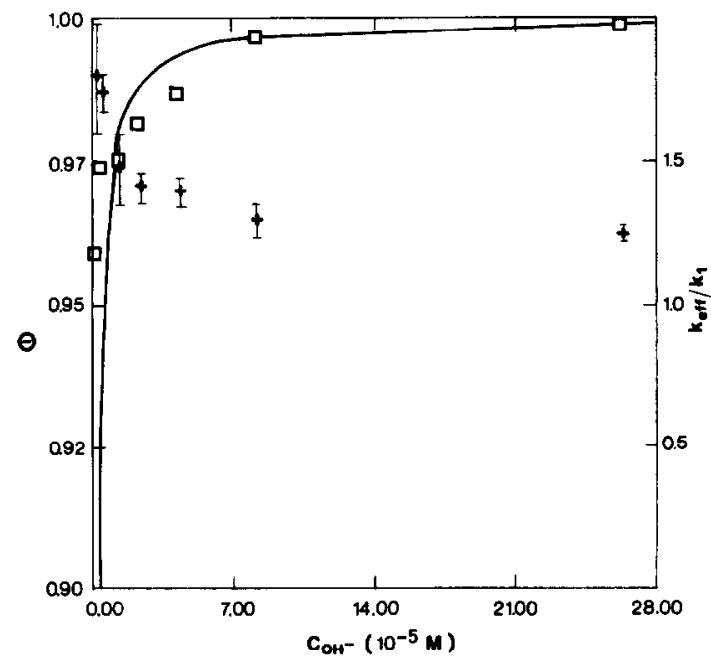

Fig. 1. Adsorption isotherm of $\mathrm{OH}^{-}$ions on colloidal ferric hydrous oxide particles. Colloid content of the sols is $9 \mathrm{mg} / 1 . \square$ stands for experimental points of the isotherm, the solid line corresponds to a Langmuir isotherm with parameters $c^{\text {adsmax }}=1.4 \times 10^{-4} \mathrm{M}$ and $K=3.6 \times 10^{6}$ $\mathrm{M}^{-1}$. The relative value of effective rate constant, $k_{\mathrm{eff}} / k_{\mathrm{s}}$ as a function of $c_{\mathrm{OH}^{-}}$is also displayed $(+)$. containing $9 \mathrm{mg} / \mathrm{l}$ ferric hydrous oxide $K=3.6 \times$ $10^{6} \mathrm{M}^{-1}$ and $c_{\mathrm{OH}^{-}}^{\text {adsmax }}=1.40 \times 10^{-4} \mathrm{M}$ were found.

\subsection{Impulse radiolysis experiments}

The Notre Dam Radiation Laboratory LINAC was used as the source of radiation. The DC conductivity signal after $8 \mathrm{MeV}$ electron pulse was detected by a fast conductivity cell and fed into a Biomation 8100 transient recorder. Compensation of stationary current provided the possibility to work up to $\mathrm{pH}=11.0$. Details of the setup and data treatment can be found in Ref. (7). The overall time constant of the whole system was about 2 ns. Extensive signal averaging and subtraction of oppositely biased signals made it possible to achieve drastic noise reduction and cancellation of the spurious signal due to the electron beam. The final curve at each composition and time scale was built up of 100 individual signals and had a reproducibility of better than $5 \%$. Dose in single shots never exceeded 100 rad and the decay kinetics appeared to be independent of dose below this value.

A recirculation cell was used in the measurements. To provide steady flow in the system we applied a peristaltic pump. The baseline conductivity of the sols was monitored with an ordinary flowthrough AC conductivity cell installed behind the glassy carbon "transient cell."(7)

A typical extra current vs time curve after averaging and subtraction is displayed in Fig. 2. Hydroxide ion concentration in the solution was 2.3

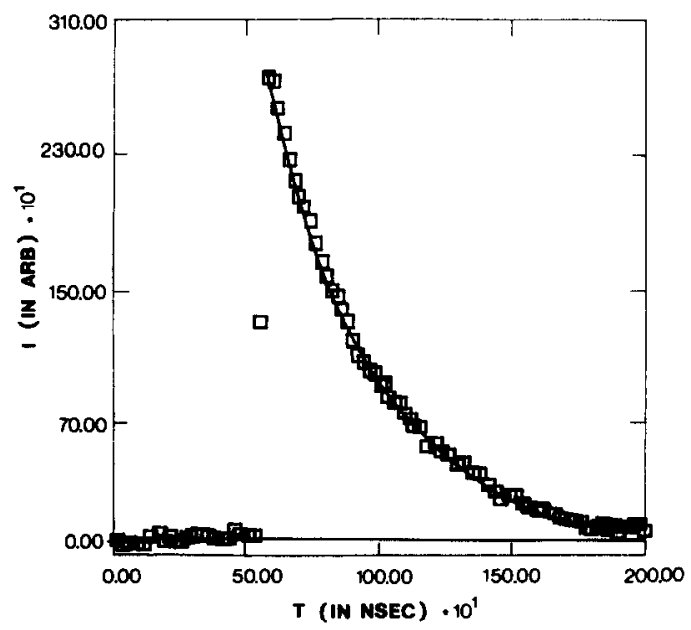

Fig. 2. Extra current vs time in colloidal ferric hydrous oxide after irradiation with $8 \mathrm{MeV}$ electrons. Experimental points ( $\square$ ) are results of averaging and substraction of oppositely biased signals. $c_{\mathrm{OH}^{-}}=2.3 \times 10^{-5} \mathrm{M}$ in the solution. Surface coverage of $98 \%$ can be obtained from the isotherm at this value. The solid line is the result of calculations based on the competitive kinetic model. 
$\times 10^{-5} \mathrm{M}$, which corresponds to $98 \%$ coverage of the colloid surface.

The order of measurements was the following. After having the freshly prepared sol aged under ultrapure $\mathbf{N}_{2}$ atmosphere the stability of the conductivity signal was checked. The first measurement was always taken at high hydroxide concentration, i.e. at the saturation part of the isotherm. Then a small amount of $0.0097 \mathrm{M} \mathrm{HCl}$ was added to the sample. Another aging of the colloid followed this until the $\mathrm{pH}$ and electric conductivity reached their stationary value. At this new equilibrium, impulse radiolysis measurements were again carried out. With this kind of titration we could follow the behavior of the system almost down to the isoelectric point between $\mathrm{pH}=8.0$ and $\mathrm{pH}=\mathbf{7 . 0}$.

\section{RESULTS AND DISCUSSION}

Pulse radiolysis of dilute alkaline solutions is well described in the literature. ${ }^{(12,13)}$ The only reaction contributing to the conductivity signal on nanosecond timescale at the given solute concentrations and dose is the neutralization reaction (I). Radiation produced hydrogen ions also react with $\mathrm{e}_{\mathrm{aq}}$ - but this reaction gives only a negligible correction to the extra current, $\Delta i$, due to the equation

$$
\Delta i \propto\left(\lambda_{\mathrm{H}^{+}}+\lambda_{\mathrm{OH}^{-}}\right) c_{\mathrm{H}^{+}}
$$$$
+\left(\lambda_{e^{-}}-\lambda_{\mathrm{OH}^{-}}\right) c_{e^{-}} .
$$

$\lambda_{\mathrm{H}^{+}}, \lambda_{\mathrm{OH}^{-}}$and $\lambda_{\mathrm{e}^{-}}$denote the equivalent conductivity of $\mathrm{H}^{+}, \mathrm{OH}^{-}$and $\mathrm{e}^{-}$. Since $\lambda_{\mathrm{e}}$ - and $\lambda_{\mathrm{OH}^{-}}$are almost equal, the second term, i.e. extra current due to solvated electrons, plays no role.

In a colloidal system, protons can react with the colloid particles as well. Although their concentration is small, each particle carries an enormous negative charge which makes them preferable reaction partners. The typical number of $\mathrm{OH}^{-}$ions in our system is $2.5-2.8 \times 10^{4}$ ions/particle, a value estimated on the basis of particle size and adsorption isotherm measurements (see 2.1). This is a consequence of specific $\mathrm{OH}^{-}$adsorption on the particle surface. Schematically the reaction can be written as follows:

$$
\begin{aligned}
& \mathrm{H}^{+}+\operatorname{coll} .\left(\mathrm{OH}^{-}\right)_{z \mathrm{coll}} \stackrel{k_{2}}{\longrightarrow} \mathrm{H}_{2} \mathrm{O} \\
&+\operatorname{coll} .\left(\mathrm{OH}^{-}\right)_{z \mathrm{coll}-1} .
\end{aligned}
$$

Competition takes place between adsorbed and bulk hydroxide ions for the radiation produced protons. The kinetic equation for the decay of $\mathrm{H}^{+}$is

$$
-\mathrm{d} c_{\mathrm{H}^{+}} / \mathrm{d} t=k_{1} c_{\mathrm{OH}^{-}}-c_{\mathrm{H}^{+}}+k_{2} c_{\mathrm{coll}} c_{\mathrm{H}^{+}},
$$

where $c_{\mathrm{H}^{+}}$and $c_{\text {coll }}$ are concentrations of the radiation produced protons and of the colloid particles, respectively.

Rewriting eqn (3):

$$
-\mathrm{d} c_{\mathrm{H}^{+}} / \mathrm{d} t=\left(k_{1}+k_{2} c_{\mathrm{coll}^{\prime}} / c_{\mathrm{OH}^{-}}\right) c_{\mathrm{H}^{+}} c_{\mathrm{OH}^{-}} .
$$

If there is no considerable change in $\mathrm{c}_{\mathrm{OH}^{-}}$during the reaction the expression in parentheses can be handled as an effective second order rate coefficient, $k_{\text {eff }}$.

We evaluated the $k_{\text {eff }} / k_{1}$ ratio from current decay after irradiation of the sols. The results as a function of $\mathrm{pH}$ are displayed in Fig. 3. Although experimental errors increase as $\mathrm{COH}^{-}$- decreases, the curve shows a significant gradient at low hydroxide concentrations. Attention is drawn to the correlation between the shape of adsorption isotherm and that of the effective rate constant as a funtion of $\mathrm{COH}^{-}$: enhancement of $k_{\text {eff }}$ and decrease from its saturation value of $\theta$ take place at similar hydroxide concentrations. This is demonstrated in Fig. 1, where the adsorption isotherm and $k_{\text {eff }} / k_{1}$ vs $c_{\mathrm{OH}^{-}}$are both displayed.

If one makes a further assumption regarding the diffusion control of both reactions (I) and (II) the ratio $k_{\text {eff }} / k_{1}$ can be expressed as

$$
\begin{aligned}
k_{\mathrm{eff}} / k_{1}= & 1+D_{\mathrm{H}}+\lambda_{\mathrm{coll}} c_{\mathrm{coll}} / \\
& \left(D_{\mathrm{H}^{+}}+D_{\mathrm{OH}^{-}}\right) \lambda \mathrm{COH}^{-},
\end{aligned}
$$

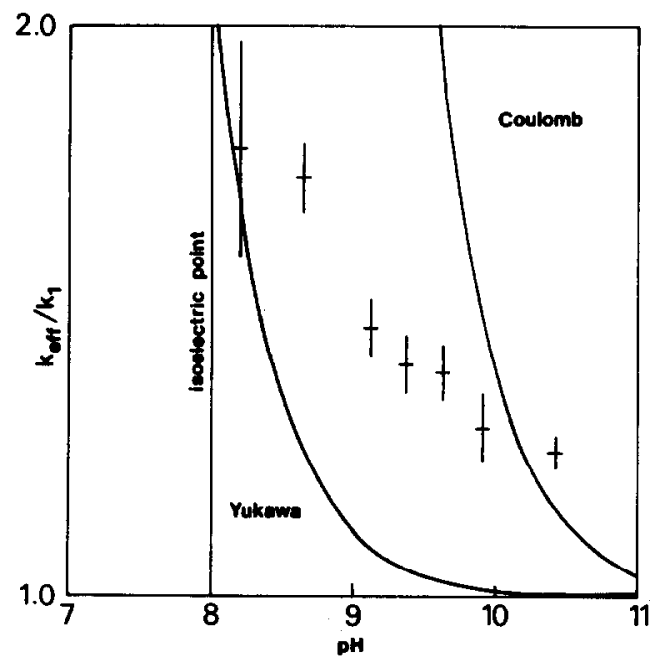

Fig. 3. Effective rate constant of neutralization reaction as a function of $\mathrm{pH}(-)$. The lower line comes from theory of diffusion controlled reactions inserting the $\mathrm{Yu}$ kawa potential for the attraction between reactants; the upper line is the result of the same theory but Coulomb potential accounts for the interaction. The isoelectric point at $\mathrm{pH} \approx 8$ hampered the measurements resulting in increased error. 
where $D_{\mathrm{H}}$ and $D_{\mathrm{OH}}$ - are the diffusion coefficients of $\mathrm{H}^{+}$and $\mathrm{OH}^{-}$ions, respectively. The definition of the so-called reaction radius $\lambda$ is

$$
\lambda^{-1}=\int_{r^{*}}^{\infty} \exp \left[V(r) / k_{B} T\right] / r^{2} \mathrm{~d} r .
$$

Here $V(r)$ denotes the interaction potential between the reactants at distance $r . r^{*}$ stands for the critical distance at which immediate reaction takes place. $\lambda_{\text {coll }}$ is the same as $\lambda$ for the reaction between a colloid particle and a proton. In eqn (5) we made use of the assumption that in reaction (II) the size of reactant coll. $\left(\mathrm{OH}^{-}\right)_{z c o l l}$ is enormous compared to an $\mathrm{OH}^{-}$ion, and consequently its diffusion can be neglected.

It is usual to apply a Coulomb potential to describe the interaction

$$
V(r)=z_{\mathrm{A}} z_{\mathrm{B}} \mathrm{e}^{2} /\left[4 \pi \epsilon_{0} \epsilon\left(r-r^{\prime}\right)\right] .
$$

$z_{\mathrm{A}}$ and $z_{\mathrm{B}}$ are the number of elementary charges $\mathrm{e}$ carried by reactants $\mathrm{A}$ and $\mathrm{B}$ and $r^{\prime}$ stands for the particle radius. For the Coulomb potential if $r^{\prime}=$ 0 the integral in eqn (6) can be evaluated analytically. Inserting the result in eqn (5) we obtain

$$
\frac{k_{\text {eff }}}{k_{1}}=1+\frac{\begin{array}{l}
D_{\mathrm{H}}+z_{\text {coll }} c_{\text {coll }} \\
\times\left(1-\exp \left[-\mathrm{e}^{2} / 4 \pi \epsilon_{0} \epsilon k_{B} T r^{*}\right]\right)
\end{array}}{\left(\begin{array}{l}
\left.D_{\mathrm{H}^{+}}+D_{\mathrm{OH}^{-}}\right) c_{\mathrm{OH}^{-}} \\
\times\left(1-\exp \left[-z_{\text {coll }} \mathrm{e}^{2} / 4 \pi \epsilon_{0} \epsilon k_{B} T r^{*}{ }_{\text {coll }}\right]\right)
\end{array}\right.}
$$

where $z_{\text {coll }}$ and $r_{\text {coll }}^{*}$, respectively, represent the number of elementary charges and the critical distance for colloid particles.

The number of elementary charges on a colloid particle is equal to the number of hydroxide ions attached to a particle

$$
z_{\mathrm{coll}}=c_{\mathrm{OH}}^{\mathrm{ads}}-/ c_{\mathrm{coll}} .
$$

Making use of eqns (1) and (9) one can evaluate eqn (8). If $r_{\text {coll }}^{*}$ is taken to be roughly equal to the radius of a colloidal particle it turns out that the exponential expression in the denominator is negligible.

Thus the $\mathrm{cOH}_{\mathrm{OH}}$-dependence of $k_{\mathrm{eff}} / k_{1}$ in the case of the Coulomb potential with $r^{\prime}=0$ is of the form

$$
\begin{aligned}
& k_{\mathrm{efff}} / k_{1}=1+D_{\mathrm{H}^{+}} K c_{\mathrm{OH}}^{\text {adsmax }} \\
& \quad \times\left(1-\exp \left[-\mathrm{e}^{2} / 4 \pi \epsilon_{0} \in k_{B} T r^{*}\right]\right) / \\
& \quad \times\left(D_{\mathrm{H}^{+}}+D_{\mathrm{OH}^{-}}\right)\left(1+K c_{\mathrm{OH}^{-}}\right)
\end{aligned}
$$

If we use the literature value $r^{*}=6.1 \AA^{(12)}$ there is no fitting parameter in eqn (10). The calculated curve is given by the upper line in Fig. 3. It is striking how this model overestimates $k_{\text {eff }} / k_{1}$ although it reflects some features of our experimental findings. The calculated $k_{\text {eff }} / k_{1}$ ratio decreases as $c_{\mathrm{OH}^{-}}$increases, reaching one as the limiting value which reminds us of the behavior of the measured $k_{\text {eff }} / k_{1}$ versus $\mathrm{C}_{\mathrm{OH}}$ - relation. No dramatic change has been observed taking $r^{\prime}$ equal to the radius of colloid particles.

This picture might be improved by the use of a more realistic interaction. The Debye-Hückel theory of electrolyte solutions shows that the interaction potential between ions is a Yukawa or screened Coulomb potential

$$
\begin{aligned}
V(r)= & z_{\mathrm{A}} z_{\mathrm{B}} \mathrm{e}^{2} \\
& \times \exp \left[-\left(r-r^{\prime}\right) / r_{D}\right] /\left[4 \pi \epsilon_{0} \epsilon\left(r-r^{\prime}\right)\right],
\end{aligned}
$$

where $r_{D}=\left(\epsilon_{0} \epsilon k_{B} T / 2 \rho N_{A} \mathrm{e}^{2} I\right)^{1 / 2}$ is the Debye screening length, $\rho$ and $I$ are the density and ionic strength of the solution, $N_{\mathrm{A}}$ denotes the Avogadro number. Phase diagram calculations of charged colloidal suspensions $^{(14)}$ also verify this potential as a form of interaction between colloid particles.

Numerical evaluation of eqn (6) in the case of the Yukawa potential yields $\lambda_{\text {coll }}$ as a function of $z_{\mathrm{col1}}$. The solid curve in Fig. 3 corresponds to eqn (5) with the screened Coulomb potential in $\lambda_{\text {cot }}$ and $\lambda$. Again, no fitting parameter has been used in this model. The dramatic change in the calculated curve shows the extreme potential sensitivity of our model. It seems quite obvious that if the attractive potential is screened it decreases the enhancement of the effective rate coefficient originating from the enormous charge carried by a colloidal particle.

Although agreement cannot be achieved either with the Coulomb or with the Yukawa potential our calculation assigns them as upper and lower limits for the real interaction.

\section{SUMMARY}

Specific absorption of hydroxide ions on the surface of ferric hydrous oxide particles in negative sols can be understood in terms of Langmuir isotherms. These particles as reactants differ in two main respects from ordinary ions, viz. their radius and charge enormously exceed those of ions.

Utilizing the example of the neutralization reaction we have demonstrated both experimentally and theoretically how the interaction potential increases along the adsorption isotherm and how this may lead to enhancement in the rate of a diffusion controlled reaction.

We measured the electric conductivity with nanosecond time resolution after an energetic electron 
pulse had produced $\mathrm{H}^{+}, \mathrm{OH}^{-}$, $\mathrm{e}_{\mathrm{aq}}^{-}$and neutral species in the system. The effective rate constant of the neutralization reaction in the sols increased with decreasing hydroxide concentration.

This finding was rationalized in terms of the theory of diffusion controlled reactions. Calculations have taught us that consideration of the Coulomb potential acting between particles overestimates the enhancement whereas results with Debye screening indicate smaller effect than observed.

Acknowledgements-The author is extremely grateful to Dr R. Schiller and to Dr R. H. Schuler for many stimulating discussions. He also wishes to express his gratitude to Dr Schuler for his invitation and hospitality. Mr T. D. Deal is kindly thanked for operating the accelerator.

\section{REFERENCES}

1. G. V. Buxton, T. Rhodes and R. M. Sellers, Nature $1982,295,583$.
2. H. S. G. Slooten and R. G. Sowden, Nature 1963 , $198,369$.

3. A. Henglein and J. Lilie, J. Am. Chem. Soc. 1981, $103,1059$.

4. D. S. Miller and G. Mclendon, J. Am. Chem. Soc. $1981,103,6791$.

5. J. Moser and M. Grätzel, Helv. Chim. Acta 1982, $65,1436$.

6. M. Grätzel, Pure Appl. Chem. 1982, 54, 2369.

7. E. Janata, Radiat. Phys. Chem. 1982, 19, 17.

8. H. B. WEISER, Inorganic Colloid Chemistry, Vol. 2, p. 51. John Wiley, New York, 1935.

9. H. Powis, J. Chem. Soc. 1915, 107, 818.

10. E. Matijevic and P. Scheiner, J. Colloid Interface Sci. 1978, 63, 509.

11. E. Matijevic, R. J. Kuo and H. Kolny, J. Colloid Interface Sci. 1981, 80, 94.

12. G. C. Barker, F. Fowles, D. C. SAmmon and B. STRINGER, Trans. Faraday Soc. 1970, 66, 1498.

13. K. D. Asmus, Int. J. Radiat. Phys. Chem. 1972, 4, 417.

14. D. Hone, S. Alexander, P. M. Chaikin and P. Pincus, J. Chem. Phys. 1983, 79, 1474. 